\title{
Intrinsic Square Function Characterizations of Variable Weak Hardy Spaces
}

\author{
Xianjie Yan
}

\begin{abstract}
Let $p(\cdot): \mathbb{R}^{n} \rightarrow(0, \infty)$ be a variable exponent function satisfying the globally log-Hölder continuous condition. In this article, via using the atomic and Littlewood-Paley function characterizations of variable weak Hardy space $W H^{p(\cdot)}\left(\mathbb{R}^{n}\right)$, the author establishes its intrinsic square function characterizations including the intrinsic Littlewood-Paley $g$-function, the intrinsic Lusin area function and the intrinsic $g_{\lambda}^{*}$-function.
\end{abstract}

\section{Introduction}

Based on the variable Lebesgue space, theories of several variable function spaces have been rapidly developed in recent years (see, for example, $3,4,10,12,24,26,37,38,40,43$, $44])$. Recall that the variable Lebesgue space $L^{p(\cdot)}\left(\mathbb{R}^{n}\right)$, with a variable exponent function $p(\cdot): \mathbb{R}^{n} \rightarrow(0, \infty)$, is a generalization of the classical Lebesgue space $L^{p}\left(\mathbb{R}^{n}\right)$, via replacing the constant exponent $p$ by the exponent function $p(\cdot)$, which consists of all functions $f$ such that $\int_{\mathbb{R}^{n}}|f(x)|^{p(x)} d x<\infty$. The study of variable Lebesgue spaces can be traced back to Birnbaum-Orlicz [7] and Orlicz [27], however, they have been the subject of more intensive study since the early 1990s because of their intrinsic interest for applications into harmonic analysis, partial differential equations and variational integrals with nonstandard growth conditions (see [8, 11, 19, 41] and their references).

Particularly, Nakai and Sawano 26 first introduced the variable Hardy spaces $H^{p(\cdot)}\left(\mathbb{R}^{n}\right)$ with $p(\cdot)$ satisfying the globally log-Hölder continuous condition, and established their atomic characterizations which were further applied to consider their duals and the boundedness of singular integral operators on $H^{p(\cdot)}\left(\mathbb{R}^{n}\right)$. Later, Sawano 29] extended the atomic characterization of $H^{p(\cdot)}\left(\mathbb{R}^{n}\right)$, which also improves the corresponding result in [26], and gave out more applications including the boundedness of several operators. Independently, Cruz-Uribe and Wang [10] also investigated the variable Hardy space $H^{p(\cdot)}\left(\mathbb{R}^{n}\right)$ with $p(\cdot)$ satisfying some conditions slightly weaker than those used in [26]. In [10], equivalent characterizations of $H^{p(\cdot)}\left(\mathbb{R}^{n}\right)$ by means of radial or non-tangential maximal functions or atoms were established. Recently, Yang et al. 42 characterized $H^{p(\cdot)}\left(\mathbb{R}^{n}\right)$ via Riesz transforms with $p(\cdot)$ satisfying the same conditions as in [10].

Received September 5, 2018; Accepted March 12, 2019.

Communicated by Duy-Minh Nhieu.

2010 Mathematics Subject Classification. Primary: 42B25; Secondary: 42B30, 42B35, 46E30.

Key words and phrases. Hardy space, variable exponent, intrinsic square function, atomic decomposition. 
It is well known that, when studying the boundedness of some operators in the critical case, the weak Hardy space $W H^{p}\left(\mathbb{R}^{n}\right)$ with $p \in(0,1]$ is a good substitute of the Hardy space $H^{p}\left(\mathbb{R}^{n}\right)$ (see $\left.\left.5,6,13,16,23,28,31,40\right]\right)$. Moreover, the space $W H^{p}\left(\mathbb{R}^{n}\right)$ was proved as the intermediate space in the real interpolation between the Hardy space $H^{p}\left(\mathbb{R}^{n}\right)$ and the space $L^{\infty}\left(\mathbb{R}^{n}\right)$ (see $\left.1,14,25,46\right)$. Motivated by these, Yan et al. [40 first introduced the variable weak Hardy spaces $W H^{p(\cdot)}\left(\mathbb{R}^{n}\right)$ via the radial grand maximal function and characterized these spaces by means of maximal functions, atoms, molecules and Littlewood-Paley functions. As an application, the authors in [40] proved the boundedness of Calderón-Zygmund operators from the variable Hardy space $H^{p(\cdot)}\left(\mathbb{R}^{n}\right)$ to $W H^{p(\cdot)}\left(\mathbb{R}^{n}\right)$ including the critical case. Very recently, Zhuo et al. [46] proved that the real interpolation between the spaces $H^{p(\cdot)}\left(\mathbb{R}^{n}\right)$ and $L^{\infty}\left(\mathbb{R}^{n}\right)$ is just $W H^{p(\cdot)}\left(\mathbb{R}^{n}\right)$ by a decomposition for any distribution of $W H^{p(\cdot)}\left(\mathbb{R}^{n}\right)$ into "good" and "bad" parts.

On the other hand, the study of the intrinsic square function on several function spaces has recently attracted many attentions. To be precise, Wilson 33 first introduced intrinsic square functions to settle a conjecture proposed by Fefferman and Stein [15] on the boundedness of the Lusin area function $S(f)$ from the weighted Lebesgue space $L_{\mathcal{M}(v)}^{2}\left(\mathbb{R}^{n}\right)$ to the weighted Lebesgue space $L_{v}^{2}\left(\mathbb{R}^{n}\right)$, where $0 \leq v \in L_{\mathrm{loc}}^{1}\left(\mathbb{R}^{n}\right)$ and $\mathcal{M}(v)$ denotes the Hardy-Littlewood maximal function of $v$. The boundedness of these intrinsic square functions on the weighted Lebesgue spaces $L_{\omega}^{p}\left(\mathbb{R}^{n}\right)$, when $p \in(1, \infty)$ and $\omega$ belongs to Muckenhoupt weights $A_{p}\left(\mathbb{R}^{n}\right)$, was proved by Wilson 34 . The intrinsic square functions, which can be thought of as "grand maximal" square functions in the style of the "grand maximal function" of Fefferman and Stein from [15], dominate all the square functions of the form $S(f)$ (and the classical ones as well), but are not essentially bigger than any one of them. Similar to the Fefferman-Stein and the Hardy-Littlewood maximal functions, their generic natures make them pointwise equivalent to each other and extremely easy to work with. Moreover, the intrinsic Lusin area function has the distinct advantage of being pointwise comparable at different cone openings, which is a property long known not to hold true for the classical Lusin area function; see Wilson [33 36] and also Lerner [20,21].

Later, Huang and Liu in 18 obtained the intrinsic square functions characterizations of the weighted Hardy space $H_{\omega}^{1}\left(\mathbb{R}^{n}\right)$ under the additional assumption that $f \in L_{\omega}^{1}\left(\mathbb{R}^{n}\right)$, which was further generalized to the weighted Hardy space, $H_{\omega}^{p}\left(\mathbb{R}^{n}\right)$ with $p \in(n /(n+$ $\alpha), 1)$ and $\alpha \in(0,1]$ by Wang and Liu in 32 , under the additional assumption that $f \in(\operatorname{Lip}(\alpha, 1,0))^{*}$, where $(\operatorname{Lip}(\alpha, 1,0))^{*}$ denotes the dual space of the Lipschitz space $\operatorname{Lip}(\alpha, 1,0)$. Recently, Liang and Yang in 22 established the $s$-order intrinsic square function characterizations of the Musielak-Orlicz Hardy space $H^{\varphi}\left(\mathbb{R}^{n}\right)$ in terms of the intrinsic Lusin area function, the intrinsic $g$-function, and the $g_{\lambda}^{*}$-function with the bestknown range $\lambda \in(2+2(\alpha+s) / n, \infty)$, which essentially improved the known results in 18 
and [32]. Very recently, Zhuo et al. [45] generalized the corresponding results in [22] to the variable Hardy space $H^{p(\cdot)}\left(\mathbb{R}^{n}\right)$ with $\lambda \in(3+2(\alpha+s) / n, \infty)$. Yan 39 generalized to the weak Musielak-Orlicz Hardy space $W H^{\varphi}\left(\mathbb{R}^{n}\right)$ with the best-known range. In addition, Wang [30] obtained the boundedness of the intrinsic square function on the weighted weak Hardy space $W H_{\omega}^{p}\left(\mathbb{R}^{n}\right)$ with $\lambda \in(3+2 \alpha / n, \infty)$. More applications of such intrinsic square functions were also given by Wilson [35,36 and Lerner [20,21].

In this article, we establish the intrinsic square function characterizations of the variable weak Hardy space $W H^{p(\cdot)}\left(\mathbb{R}^{n}\right)$ introduced by Yan et al. in [40], including the intrinsic Littlewood-Paley $g$-function, the intrinsic Lusin area function and the intrinsic $g_{\lambda}^{*}$-function by using the atomic and Littlewood-Paley function characterizations theorems of the space $W H^{p(\cdot)}\left(\mathbb{R}^{n}\right)$ obtained in 40 .

To state the results, we first make some conventions on notation. Let $\mathbb{N}:=\{1,2, \ldots\}$ and $\mathbb{Z}_{+}:=\mathbb{N} \cup\{0\}$. We denote by $C$ a positive constant which is independent of the main parameters, but may vary from line to line. We use $C_{(\alpha, \ldots)}$ to denote a positive constant depending on the indicated parameters $\alpha, \ldots$. The symbol $A \lesssim B$ means $A \leq C B$. If $A \lesssim B$ and $B \lesssim A$, we then write $A \sim B$. If $E$ is a subset of $\mathbb{R}^{n}$, we denote by $\chi_{E}$ its characteristic function and by $E^{\complement}$ the set $\mathbb{R}^{n} \backslash E$. For all $r \in(0, \infty)$ and $x \in \mathbb{R}^{n}$, denote by $B(x, r)$ the ball centered at $x$ with the radius $r$, namely, $B(x, r):=\left\{y \in \mathbb{R}^{n}:|x-y|<r\right\}$. For any ball $B$, we use $x_{B}$ to denote its center and $r_{B}$ its radius, and denote by $\lambda B$ for any $\lambda \in(0, \infty)$ the ball concentric with $B$ having the radius $\lambda r_{B}$.

We also recall some notation about variable Lebesgue spaces. For an exposition of these concepts, we refer the reader to the monographs [8,11]. A measurable function $p(\cdot): \mathbb{R}^{n} \rightarrow(0, \infty)$ is called a variable exponent. Denote by $\mathcal{P}\left(\mathbb{R}^{n}\right)$ the collection of all variable exponents $p(\cdot)$ satisfying

$$
0<p_{-}:=\operatorname{essinf}_{x \in \mathbb{R}^{n}} p(x) \leq \underset{x \in \mathbb{R}^{n}}{\operatorname{ess} \sup } p(x)=: p_{+}<\infty .
$$

For a measurable function $f$ on $\mathbb{R}^{n}$ and $p(\cdot) \in \mathcal{P}\left(\mathbb{R}^{n}\right)$, the modular functional (or, simply, the modular $) \varrho_{p(\cdot)}$, associated with $p(\cdot)$, is defined by setting

$$
\varrho_{p(\cdot)}(f):=\int_{\mathbb{R}^{n}}|f(x)|^{p(x)} d x
$$

and the Luxemburg (also known as the Luxemburg-Nakano) quasi-norm is given by setting

$$
\|f\|_{L^{p(\cdot)\left(\mathbb{R}^{n}\right)}}:=\inf \left\{\lambda \in(0, \infty): \varrho_{p(\cdot)}(f / \lambda) \leq 1\right\}
$$

Definition 1.1. Let $p(\cdot) \in \mathcal{P}\left(\mathbb{R}^{n}\right)$.

(i) The variable Lebesgue space $L^{p(\cdot)}\left(\mathbb{R}^{n}\right)$ is defined to be the set of all measurable functions $f$ such that $\|f\|_{L^{p(\cdot)}\left(\mathbb{R}^{n}\right)}<\infty$. 
(ii) The variable weak Lebesgue space $W L^{p(\cdot)}\left(\mathbb{R}^{n}\right)$ is defined to be the set of all measurable functions $f$ such that

$$
\|f\|_{W L^{p(\cdot)\left(\mathbb{R}^{n}\right)}}:=\sup _{\alpha \in(0, \infty)} \alpha\left\|\chi_{\left\{x \in \mathbb{R}^{n}:|f(x)|>\alpha\right\}}\right\|_{L^{p(\cdot)}\left(\mathbb{R}^{n}\right)}<\infty .
$$

Remark 1.2. Let $p(\cdot) \in \mathcal{P}\left(\mathbb{R}^{n}\right)$ and $f \in L^{p(\cdot)}\left(\mathbb{R}^{n}\right)$.

(i) $\|f\|_{L^{p(\cdot)}\left(\mathbb{R}^{n}\right)} \geq 0$, and $\|f\|_{L^{p(\cdot)}\left(\mathbb{R}^{n}\right)}=0$ if and only if $f(x)=0$ for almost every $x \in \mathbb{R}^{n}$.

(ii) For all $\lambda \in \mathbb{C},\|\lambda f\|_{L^{p(\cdot)\left(\mathbb{R}^{n}\right)}}=|\lambda|\|f\|_{L^{p(\cdot)\left(\mathbb{R}^{n}\right)}}$.

(iii) For all $s \in(0, \infty)$,

$$
\left\||f|^{s}\right\|_{L^{p(\cdot)}\left(\mathbb{R}^{n}\right)}=\|f\|_{L^{s p(\cdot)}\left(\mathbb{R}^{n}\right)}^{s}
$$

Moreover, for all $\ell \in(0, \underline{p}]$,

$$
\|f+g\|_{L^{p(\cdot)}\left(\mathbb{R}^{n}\right)}^{\ell} \leq\|f\|_{L^{p(\cdot)\left(\mathbb{R}^{n}\right)}}^{\ell}+\|g\|_{L^{p(\cdot)}\left(\mathbb{R}^{n}\right)}^{\ell},
$$

here and hereafter,

$$
\underline{p}:=\min \left\{p_{-}, 1\right\}
$$

with $p_{-}$as in (1.1). Particularly, when $p_{-} \in[1, \infty), L^{p(\cdot)}\left(\mathbb{R}^{n}\right)$ is a Banach space (see [11, Theorem 3.2.7]).

The following Fatou lemma of $L^{p(\cdot)}\left(\mathbb{R}^{n}\right)$ was obtained in 8 , Theorem 2.61].

Lemma 1.3. Let $p(\cdot): \mathbb{R}^{n} \rightarrow[1, \infty)$ and $\left\{f_{k}\right\}_{k \in \mathbb{N}} \subset L^{p(\cdot)}\left(\mathbb{R}^{n}\right)$. If $f_{k} \rightarrow f$ as $k \rightarrow \infty$ pointwise almost everywhere in $\mathbb{R}^{n}$ and $\liminf _{k \rightarrow \infty}\left\|f_{k}\right\|_{L^{p(\cdot)}\left(\mathbb{R}^{n}\right)}$ is finite, then $f \in L^{p(\cdot)}\left(\mathbb{R}^{n}\right)$ and

$$
\|f\|_{L^{p(\cdot)\left(\mathbb{R}^{n}\right)}} \leq \liminf _{k \rightarrow \infty}\left\|f_{k}\right\|_{L^{p(\cdot)\left(\mathbb{R}^{n}\right)}} .
$$

A function $p(\cdot) \in \mathcal{P}\left(\mathbb{R}^{n}\right)$ is said to satisfy the globally log-Hölder continuous condition, denoted by $p(\cdot) \in C^{\log }\left(\mathbb{R}^{n}\right)$, if there exist positive constants $C_{\log }(p)$ and $C_{\infty}$, and $p_{\infty} \in \mathbb{R}$ such that, for all $x, y \in \mathbb{R}^{n}$,

$$
|p(x)-p(y)| \leq \frac{C_{\log }(p)}{\log (e+1 /|x-y|)}
$$

and

$$
\left|p(x)-p_{\infty}\right| \leq \frac{C_{\infty}}{\log (e+|x|)}
$$

In what follows, denote by $\mathcal{S}\left(\mathbb{R}^{n}\right)$ the space of all Schwartz functions and $\mathcal{S}^{\prime}\left(\mathbb{R}^{n}\right)$ its topological dual space equipped with the weak-* topology. For any $N \in \mathbb{N}$, let

$$
\mathcal{F}_{N}\left(\mathbb{R}^{n}\right):=\left\{\psi \in \mathcal{S}\left(\mathbb{R}^{n}\right): \sum_{\beta \in \mathbb{Z}_{+}^{n},|\beta| \leq N} \sup _{x \in \mathbb{R}^{n}}\left[(1+|x|)^{N}\left|D^{\beta} \psi(x)\right|\right] \leq 1\right\}
$$


where, for any $\beta:=\left(\beta_{1}, \ldots, \beta_{n}\right) \in \mathbb{Z}_{+}^{n},|\beta|:=\beta_{1}+\cdots+\beta_{n}$ and $D^{\beta}:=\left(\frac{\partial}{\partial x_{1}}\right)^{\beta_{1}} \cdots\left(\frac{\partial}{\partial x_{n}}\right)^{\beta_{n}}$. Then, for all $f \in \mathcal{S}^{\prime}\left(\mathbb{R}^{n}\right)$, the radial grand maximal function $f_{N,+}^{*}$ of $f$ is defined by setting, for all $x \in \mathbb{R}^{n}$,

$$
f_{N,+}^{*}(x):=\sup \left\{\left|f * \psi_{t}(x)\right|: t \in(0, \infty) \text { and } \psi \in \mathcal{F}_{N}\left(\mathbb{R}^{n}\right)\right\}
$$

here and hereafter, for all $t \in(0, \infty), \psi_{t}(\cdot):=t^{-n} \psi(\cdot / t)$.

For any measurable set $E \subset \mathbb{R}^{n}$ and $r \in(0, \infty)$, let $L^{r}(E)$ be the set of all measurable functions $f$ such that

$$
\|f\|_{L^{r}(E)}:=\left[\int_{E}|f(x)|^{r} d x\right]^{1 / r}<\infty .
$$

For $r \in(0, \infty)$, denote by $L_{\text {loc }}^{r}\left(\mathbb{R}^{n}\right)$ the set of all $r$-locally integrable functions on $\mathbb{R}^{n}$. Recall that the Hardy-Littlewood maximal operator $\mathcal{M}$ is defined by setting, for all $f \in L_{\text {loc }}^{1}\left(\mathbb{R}^{n}\right)$ and $x \in \mathbb{R}^{n}$,

$$
\mathcal{M}(f)(x):=\sup _{B \ni x} \frac{1}{|B|} \int_{B}|f(y)| d y,
$$

where the supremum is taken over all balls $B$ of $\mathbb{R}^{n}$ containing $x$.

Now we recall the definitions of the variable Hardy space (see [26, 45]) and the variable weak Hardy space (see 40,46$]$ ).

Definition 1.4. Let $p(\cdot) \in C^{\log }\left(\mathbb{R}^{n}\right)$ and $N \in(n / \underline{p}+n+1, \infty)$ be a positive integer, where $\underline{p}$ is as in 1.2 .

(i) The variable Hardy space $H^{p(\cdot)}\left(\mathbb{R}^{n}\right)$ is defined to be the set of all $f \in \mathcal{S}^{\prime}\left(\mathbb{R}^{n}\right)$ such that $f_{N,+}^{*} \in L^{p(\cdot)}\left(\mathbb{R}^{n}\right)$, equipped with the quasi-norm

$$
\|f\|_{H^{p(\cdot)}\left(\mathbb{R}^{n}\right)}:=\left\|f_{N,+}^{*}\right\|_{L^{p(\cdot)}\left(\mathbb{R}^{n}\right)} \cdot
$$

(ii) The variable weak Hardy space $W H^{p(\cdot)}\left(\mathbb{R}^{n}\right)$ is defined to be the set of all $f \in \mathcal{S}^{\prime}\left(\mathbb{R}^{n}\right)$ such that $f_{N,+}^{*} \in W L^{p(\cdot)}\left(\mathbb{R}^{n}\right)$, equipped with the quasi-norm

$$
\|f\|_{W H^{p(\cdot)\left(\mathbb{R}^{n}\right)}}:=\left\|f_{N,+}^{*}\right\|_{W L^{p(\cdot)\left(\mathbb{R}^{n}\right)}} \cdot
$$

Remark 1.5. (i) The spaces $H^{p(\cdot)}\left(\mathbb{R}^{n}\right)$ and $W H^{p(\cdot)}\left(\mathbb{R}^{n}\right)$ are independent of the choice of $N \in(n / \underline{p}+n+1, \infty)$, see [26, Theorem 3.3] and [40, Remark 2.14(i)].

(ii) If $p(\cdot) \equiv p \in(0, \infty)$, the spaces $H^{p(\cdot)}\left(\mathbb{R}^{n}\right)$ and $W H^{p(\cdot)}\left(\mathbb{R}^{n}\right)$ are, respectively, classical Hardy space $H^{p}\left(\mathbb{R}^{n}\right)$ and classical weak Hardy space $W H^{p}\left(\mathbb{R}^{n}\right)$. 
For any $s \in \mathbb{Z}_{+}, C^{s}\left(\mathbb{R}^{n}\right)$ denotes the set of all functions having continuous classical derivatives up to an order of not more than $s$. For $\alpha \in(0,1]$ and $s \in \mathbb{Z}_{+}$, let $\mathcal{C}_{\alpha, s}\left(\mathbb{R}^{n}\right)$ be the set of functions $\phi \in C^{s}\left(\mathbb{R}^{n}\right)$ such that $\operatorname{supp} \phi \subset\left\{x \in \mathbb{R}^{n}:|x| \leq 1\right\}, \int_{\mathbb{R}^{n}} \phi(x) x^{\gamma} d x=0$ for all $\gamma \in \mathbb{Z}_{+}^{n}$ and $|\gamma| \leq s$, and, for all $x_{1}, x_{2} \in \mathbb{R}^{n}$ and $\nu \in \mathbb{Z}_{+}^{n}$ with $|\nu|=s$,

$$
\left|D^{\nu} \phi\left(x_{1}\right)-D^{\nu} \phi\left(x_{2}\right)\right| \leq\left|x_{1}-x_{2}\right|^{\alpha}
$$

For all $f \in L_{\mathrm{loc}}^{1}\left(\mathbb{R}^{n}\right)$ and $(y, t) \in \mathbb{R}_{+}^{n+1}:=\mathbb{R}^{n} \times(0, \infty)$, let

$$
A_{\alpha, s}(f)(y, t):=\sup _{\phi \in \mathcal{C}_{\alpha, s}\left(\mathbb{R}^{n}\right)}\left|f * \phi_{t}(y)\right|
$$

Then, the intrinsic $g$-function, the intrinsic Lusin area integral, and the intrinsic $g_{\lambda}^{*}$ function of $f$ are, respectively, defined by setting, for all $x \in \mathbb{R}^{n}$ and $\lambda \in(0, \infty)$,

$$
\begin{aligned}
g_{\alpha, s}(f)(x) & :=\left\{\int_{0}^{\infty}\left[A_{\alpha, s}(f)(x, t)\right]^{2} \frac{d t}{t}\right\}^{1 / 2}, \\
S_{\alpha, s}(f)(x) & :=\left\{\int_{\Gamma(x)}\left[A_{\alpha, s}(f)(y, t)\right]^{2} \frac{d y d t}{t^{n+1}}\right\}^{1 / 2}
\end{aligned}
$$

and

$$
g_{\lambda, \alpha, s}^{*}(f)(x):=\left\{\int_{0}^{\infty} \int_{\mathbb{R}^{n}}\left(\frac{t}{t+|x-y|}\right)^{\lambda n}\left[A_{\alpha, s}(f)(y, t)\right]^{2} \frac{d y d t}{t^{n+1}}\right\}^{1 / 2}
$$

here and hereafter, $\Gamma(x):=\left\{(y, t) \in \mathbb{R}_{+}^{n+1}:|y-x|<t\right\}$.

We also recall another kind of similar-looking square functions, defined via convolutions with kernels that have unbounded supports. For $\alpha \in(0,1], s \in \mathbb{Z}_{+}$and $\epsilon \in(0, \infty)$, let $\mathcal{C}_{(\alpha, \epsilon), s}\left(\mathbb{R}^{n}\right)$ be the set of functions $\phi \in C^{s}\left(\mathbb{R}^{n}\right)$ such that, for all $x \in \mathbb{R}^{n}, \gamma \in \mathbb{Z}_{+}^{n}$ and $|\gamma| \leq s,\left|D^{\gamma} \phi(x)\right| \leq(1+|x|)^{-n-\epsilon}, \int_{\mathbb{R}^{n}} \phi(x) x^{\gamma} d x=0$ and, for all $x_{1}, x_{2} \in \mathbb{R}^{n}, \nu \in \mathbb{Z}_{+}^{n}$ with $|\nu|=s$,

$$
\left|D^{\nu} \phi\left(x_{1}\right)-D^{\nu} \phi\left(x_{2}\right)\right| \leq\left|x_{1}-x_{2}\right|^{\alpha}\left[\left(1+\left|x_{1}\right|\right)^{-n-\epsilon}+\left(1+\left|x_{2}\right|\right)^{-n-\epsilon}\right]
$$

Remark that, in what follows, the parameter $\epsilon$ usually has to be chosen to be large enough. For all $f$ satisfying

$$
|f(\cdot)|(1+|\cdot|)^{-n-\epsilon} \in L^{1}\left(\mathbb{R}^{n}\right)
$$

and $(y, t) \in \mathbb{R}_{+}^{n+1}$, let

$$
\widetilde{A}_{(\alpha, \epsilon), s}(f)(y, t):=\sup _{\phi \in \mathcal{C}_{(\alpha, \epsilon), s}\left(\mathbb{R}^{n}\right)}\left|f * \phi_{t}(y)\right| .
$$


Then, for all $x \in \mathbb{R}^{n}$ and $\lambda \in(0, \infty)$, we let

$$
\begin{aligned}
\widetilde{g}_{(\alpha, \epsilon), s}(f)(x) & :=\left\{\int_{0}^{\infty}\left[\widetilde{A}_{(\alpha, \epsilon), s}(f)(x, t)\right]^{2} \frac{d t}{t}\right\}^{1 / 2} \\
\widetilde{S}_{(\alpha, \epsilon), s}(f)(x) & :=\left\{\int_{\Gamma(x)}\left[\widetilde{A}_{(\alpha, \epsilon), s}(f)(y, t)\right]^{2} \frac{d y d t}{t^{n+1}}\right\}^{1 / 2}
\end{aligned}
$$

and

$$
\widetilde{g}_{\lambda,(\alpha, \epsilon), s}^{*}(f)(x):=\left\{\int_{0}^{\infty} \int_{\mathbb{R}^{n}}\left(\frac{t}{t+|x-y|}\right)^{\lambda n}\left[\widetilde{A}_{(\alpha, \epsilon), s}(f)(y, t)\right]^{2} \frac{d y d t}{t^{n+1}}\right\}^{1 / 2} .
$$

These intrinsic square functions, when $s=0$, were originally introduced by Wilson [33], which were further generalized to $s \in \mathbb{Z}_{+}$by Liang and Yang [22].

Recall that $f \in \mathcal{S}^{\prime}\left(\mathbb{R}^{n}\right)$ is said to vanish weakly at infinity if, for every $\phi \in \mathcal{S}\left(\mathbb{R}^{n}\right)$, $f * \phi_{t} \rightarrow 0$ in $\mathcal{S}^{\prime}\left(\mathbb{R}^{n}\right)$ as $t \rightarrow \infty$ (see, for example, [17, p. 50]).

Now we state the main results of this article.

Theorem 1.6. Let $p(\cdot) \in C^{\log }\left(\mathbb{R}^{n}\right)$ and $p_{+} \in(0,1]$. Assume that $\alpha \in(0,1], s \in \mathbb{Z}_{+}$and $p_{-} \in(n /(n+\alpha+s), 1]$.

(i) If $f \in \mathcal{S}^{\prime}\left(\mathbb{R}^{n}\right), f$ vanishes weakly at infinity and $g_{\alpha, s}(f) \in W L^{p(\cdot)}\left(\mathbb{R}^{n}\right)$, then $f \in$ $W H^{p(\cdot)}\left(\mathbb{R}^{n}\right)$, moreover, there exists a positive constant $C$, independent of $f$, such that

$$
\|f\|_{W H^{p(\cdot)}\left(\mathbb{R}^{n}\right)} \leq C\left\|g_{\alpha, s}(f)\right\|_{W L^{p(\cdot)\left(\mathbb{R}^{n}\right)}} ;
$$

(ii) If $f \in\left(\mathcal{C}_{(\alpha, \epsilon), s}\left(\mathbb{R}^{n}\right)\right)^{*}$, with $\left(\mathcal{C}_{(\alpha, \epsilon), s}\left(\mathbb{R}^{n}\right)\right)^{*}$ being the dual space of $\mathcal{C}_{(\alpha, \epsilon), s}\left(\mathbb{R}^{n}\right)$, then there exists a positive constant $C$ such that, for all $f \in W H^{p(\cdot)}\left(\mathbb{R}^{n}\right)$, it holds true that

$$
\left\|g_{\alpha, s}(f)\right\|_{W L^{p(\cdot)}\left(\mathbb{R}^{n}\right)} \leq C\|f\|_{W H^{p(\cdot)}\left(\mathbb{R}^{n}\right)} .
$$

The same is true if $g_{\alpha, s}(f)$ is replaced, respectively, by $S_{\alpha, s}(f), \widetilde{g}_{(\alpha, \epsilon), s}(f)$ and $\widetilde{S}_{(\alpha, \epsilon), s}(f)$ with $\epsilon \in(\max \{\alpha, s\}, \infty)$.

Theorem 1.7. Let $p(\cdot) \in C^{\log }\left(\mathbb{R}^{n}\right)$ and $p_{+} \in(0,1]$. Assume that $\alpha \in(0,1], s \in \mathbb{Z}_{+}$, $p_{-} \in(n /(n+\alpha+s), 1]$ and $\lambda \in(3+2(\alpha+s) / n, \infty)$.

(i) If $f \in \mathcal{S}^{\prime}\left(\mathbb{R}^{n}\right)$, $f$ vanishes weakly at infinity and $g_{\lambda, \alpha, s}^{*}(f) \in W L^{p(\cdot)}\left(\mathbb{R}^{n}\right)$, then $f \in$ $W H^{p(\cdot)}\left(\mathbb{R}^{n}\right)$, moreover, there exists a positive constant $C$, independent of $f$, such that

$$
\|f\|_{W H^{p(\cdot)\left(\mathbb{R}^{n}\right)}} \leq C\left\|g_{\lambda, \alpha, s}^{*}(f)\right\|_{W L^{p(\cdot)\left(\mathbb{R}^{n}\right)}}
$$

(ii) If $f \in\left(\mathcal{C}_{(\alpha, \epsilon), s}\left(\mathbb{R}^{n}\right)\right)^{*}$, then there exists a positive constant $C$ such that, for all $f \in$ $W H^{p(\cdot)}\left(\mathbb{R}^{n}\right)$, it holds true that

$$
\left\|g_{\lambda, \alpha, s}^{*}(f)\right\|_{W L^{p(\cdot)}\left(\mathbb{R}^{n}\right)} \leq C\|f\|_{W H^{p(\cdot)\left(\mathbb{R}^{n}\right)}}
$$


The same is true if $g_{\lambda, \alpha, s}^{*}(f)$ is replaced by $\widetilde{g}_{\lambda,(\alpha, \epsilon), s}^{*}(f)$ with $\epsilon \in(\max \{\alpha, s\}, \infty)$.

Remark 1.8. (i) We point out that there exists a positive constant $C$ such that, for all $\phi \in \mathcal{C}_{\alpha, s}\left(\mathbb{R}^{n}\right), C \phi \in \mathcal{C}_{(\alpha, \epsilon), s}\left(\mathbb{R}^{n}\right)$ and hence the intrinsic square functions are well defined for functionals in $\left(\mathcal{C}_{(\alpha, \epsilon), s}\left(\mathbb{R}^{n}\right)\right)^{*}$.

(ii) Recall that Wang [30 obtained the boundedness of the intrinsic square functions on the weighted weak Hardy space $W H_{w}^{p}\left(\mathbb{R}^{n}\right)$. Moreover, for $\alpha \in(0,1], p \in(n /(n+\alpha), 1]$ and $w \in A_{p(1+\alpha / n)}\left(\mathbb{R}^{n}\right)$, Wang $[30$ ] established the boundedness of the intrinsic Littlewood-Paley $g_{\lambda}^{*}$ function with $\lambda \in(3+2 \alpha / n, \infty)$, which coincides with the case when $s=0$ and $p(x) \equiv p$ for all $x \in \mathbb{R}^{n}$ of Theorem 1.7 (ii).

(iii) Recently, Yan [39] characterized the weak Musielak-Orlicz Hardy space $W H^{\varphi}\left(\mathbb{R}^{n}\right)$ in terms of the intrinsic Littlewood-Paley $g_{\lambda}^{*}$-functions with $\lambda \in(2+2(\alpha+s) / n, \infty)$, which coincides with the best known range of the $g_{\lambda}^{*}$-functions characterizations for the classical Hardy space $H^{p}\left(\mathbb{R}^{n}\right.$ ) (see, for example, 2, 17]). However, as was mentioned in 45 , Remark 1.11(ii)], it is unclear whether the intrinsic $g_{\lambda}^{*}$-functions, when $\lambda \in(2+2(\alpha+s) / n, 3+2(\alpha+s) / n)$, can characterize the space $W H^{p(\cdot)}\left(\mathbb{R}^{n}\right)$ or not, since the method used in the proof of [39, Theorem 1.7] strongly depends on the properties of uniformly Muckenhoupt weights.

This article is organized as follows. Section 2 is devoted to the proofs of Theorems 1.6 and 1.7. The key tools used to prove Theorem 1.6 are the fact that the intrinsic square functions are pointwise comparable as was proved in 22] (see also Lemmas 2.8 and 2.9 below), the atomic decomposition and Littlewood-Paley $g$-function characterizations of $W H^{p(\cdot)}\left(\mathbb{R}^{n}\right)$ established in $[40]$ (see also Propositions 2.1 and 2.2 below). As an application of Theorem 1.6, we give the proof of Theorem 1.7 by showing that, for all $x \in \mathbb{R}^{n}$, the intrinsic square functions $\widetilde{g}_{\lambda,(\alpha, \epsilon), s}^{*}(f)(x)$ and $\widetilde{S}_{(\alpha, \epsilon), s}(f)(x)$ are pointwise comparable under the assumption $\lambda \in(3+2(\alpha+s) / n, \infty)$.

\section{Proofs of main results}

We first recall the variable weak atomic Hardy space introduced by Yan [40]. Let $p(\cdot) \in$ $C^{\log }\left(\mathbb{R}^{n}\right), q \in(1, \infty]$ and $s \in\left(n / p_{-}-n-1, \infty\right) \cap \mathbb{Z}_{+}$with $p_{-}$as in 1.1). A measurable function $a$ on $\mathbb{R}^{n}$ is called a $(p(\cdot), q, s)$-atom if there exists a ball $B$ such that

(i) $\operatorname{supp} a \subset B$;

(ii) $\|a\|_{L^{q}\left(\mathbb{R}^{n}\right)} \leq \frac{|B|^{1 / q}}{\left\|\chi_{B}\right\|_{L^{p(\cdot)}\left(\mathbb{R}^{n}\right)}} ;$

(iii) $\int_{\mathbb{R}^{n}} a(x) x^{\alpha} d x=0$ for all $\alpha \in \mathbb{Z}_{+}^{n}$ with $|\alpha| \leq s$. 
The variable weak atomic Hardy space, denoted by $W H_{\mathrm{atom}}^{p(\cdot), q, s}\left(\mathbb{R}^{n}\right)$, is defined as the space of all $f \in \mathcal{S}^{\prime}\left(\mathbb{R}^{n}\right)$ which can be decomposed as

$$
f=\sum_{i \in \mathbb{Z}} \sum_{j \in \mathbb{N}} \lambda_{i, j} a_{i, j} \quad \text { in } \mathcal{S}^{\prime}\left(\mathbb{R}^{n}\right)
$$

where $\left\{a_{i, j}\right\}_{i \in \mathbb{Z}, j \in \mathbb{N}}$ is a sequence of $(p(\cdot), q, s)$-atoms, associated with balls $\left\{B_{i, j}\right\}_{i \in \mathbb{Z}, j \in \mathbb{N}}$, satisfying that there exists a positive constant $c \in(0,1]$ such that, for all $x \in \mathbb{R}^{n}$ and $i \in \mathbb{Z}$, $\sum_{j \in \mathbb{N}} \chi_{c B_{i, j}}(x) \leq C$ with $C$ being a positive constant independent of $x$ and $i$ and, for all $i \in \mathbb{Z}$ and $j \in \mathbb{N}, \lambda_{i, j}:=\widetilde{C} 2^{i}\left\|\chi_{B_{i, j}}\right\|_{L^{p(\cdot)}\left(\mathbb{R}^{n}\right)}$ with $\widetilde{C}$ being a positive constant independent of $i$ and $j$.

Moreover, for any $f \in W H_{\text {atom }}^{p(\cdot), q, s}\left(\mathbb{R}^{n}\right)$, define

$$
\|f\|_{W H_{\mathrm{atom}}^{p(\cdot), q, s}\left(\mathbb{R}^{n}\right)}:=\inf \left[\sup _{i \in \mathbb{Z}}\left\|\left\{\sum_{j \in \mathbb{N}}\left[\frac{\lambda_{i, j} \chi_{B_{i, j}}}{\left\|\chi_{B_{i, j}}\right\|_{L^{p(\cdot)}\left(\mathbb{R}^{n}\right)}}\right]^{\underline{p}}\right\}^{1 / \underline{p}}\right\|_{L^{p(\cdot)}\left(\mathbb{R}^{n}\right)}\right],
$$

where the infimum is taken over all decompositions of $f$ as above.

Proposition 2.1. Let $p(\cdot) \in C^{\log }\left(\mathbb{R}^{n}\right), s \in\left(n / p_{-}-n-1, \infty\right) \cap \mathbb{Z}_{+}$and $q \in\left(\max \left\{p_{+}, 1\right\}, \infty\right]$ with $p_{-}$and $p_{+}$as in (1.1). Then $W H^{p(\cdot)}\left(\mathbb{R}^{n}\right)=W H_{\text {atom }}^{p(\cdot), q, s}\left(\mathbb{R}^{n}\right)$ with equivalent quasinorms.

Let $\phi \in \mathcal{S}\left(\mathbb{R}^{n}\right)$ be a radial function satisfying

$$
\begin{aligned}
& \operatorname{supp} \phi \subset\left\{x \in \mathbb{R}^{n}:|x| \leq 1\right\}, \\
& \int_{\mathbb{R}^{n}} \phi(x) x^{\gamma} d x=0 \quad \text { for all } \gamma \in \mathbb{Z}_{+}^{n} \text { with }|\gamma| \leq \max \left\{\left\lfloor\frac{n}{p_{-}}-n-1\right\rfloor, 0\right\}
\end{aligned}
$$

and

$$
\int_{0}^{\infty}|\widehat{\phi}(\xi t)|^{2} \frac{d t}{t}=1 \quad \text { for all } \xi \in \mathbb{R}^{n} \backslash\left\{\overrightarrow{0}_{n}\right\} .
$$

Recall that, for all $f \in \mathcal{S}^{\prime}\left(\mathbb{R}^{n}\right)$, the Littlewood-Paley $g$-function and the $g_{\lambda}^{*}$-function of $f$ with $\lambda \in(0, \infty)$ are defined, respectively, by setting, for all $x \in \mathbb{R}^{n}$,

$$
g(f)(x):=\left[\int_{0}^{\infty}\left|f * \phi_{t}(x)\right|^{2} \frac{d t}{t}\right]^{1 / 2}
$$

and

$$
g_{\lambda}^{*}(f)(x):=\left[\int_{0}^{\infty} \int_{\mathbb{R}^{n}}\left(\frac{t}{t+|x-y|}\right)^{\lambda n}\left|f * \phi_{t}(y)\right|^{2} \frac{d y d t}{t^{n+1}}\right]^{1 / 2} .
$$

The following conclusions are just 40, Theorems 6.2 and 6.3]. 
Proposition 2.2. Let $p(\cdot) \in C^{\log }\left(\mathbb{R}^{n}\right)$. Then $f \in W H^{p(\cdot)}\left(\mathbb{R}^{n}\right)$ if and only if $f \in \mathcal{S}^{\prime}\left(\mathbb{R}^{n}\right)$, $f$ vanishes weakly at infinity and $g(f) \in W L^{p(\cdot)}\left(\mathbb{R}^{n}\right)$. Moreover, for all $f \in W H^{p(\cdot)}\left(\mathbb{R}^{n}\right)$,

$$
C^{-1}\|g(f)\|_{W L^{p(\cdot)\left(\mathbb{R}^{n}\right)}} \leq\|f\|_{W H^{p(\cdot)}\left(\mathbb{R}^{n}\right)} \leq C\|g(f)\|_{W L^{p(\cdot)\left(\mathbb{R}^{n}\right)}},
$$

where $C$ is a positive constant independent of $f$.

Proposition 2.3. Let $p(\cdot) \in C^{\log }\left(\mathbb{R}^{n}\right)$ and $\lambda \in\left(1+\frac{2}{\min \left\{p_{-}, 2\right\}}, \infty\right)$. Then $f \in W H^{p(\cdot)}\left(\mathbb{R}^{n}\right)$ if and only if $f \in \mathcal{S}^{\prime}\left(\mathbb{R}^{n}\right), f$ vanishes weakly at infinity and $g_{\lambda}^{*}(f) \in W L^{p(\cdot)}\left(\mathbb{R}^{n}\right)$. Moreover, for all $f \in W H^{p(\cdot)}\left(\mathbb{R}^{n}\right)$,

$$
C^{-1}\left\|g_{\lambda}^{*}(f)\right\|_{W L^{p(\cdot)}\left(\mathbb{R}^{n}\right)} \leq\|f\|_{W H^{p(\cdot)}\left(\mathbb{R}^{n}\right)} \leq C\left\|g_{\lambda}^{*}(f)\right\|_{W L^{p(\cdot)}\left(\mathbb{R}^{n}\right)},
$$

where $C$ is a positive constant independent of $f$.

To prove Theorems 1.6 and 1.7, we need more preparations. The following FeffermanStein vector-valued inequality of the Hardy-Littlewood maximal operator $\mathcal{M}$ on the variable Lebesgue space $L^{p(\cdot)}\left(\mathbb{R}^{n}\right)$ was obtained in 9 , Corollary 2.1].

Lemma 2.4. Let $r \in(1, \infty)$. Assume that $p(\cdot) \in C^{\log }\left(\mathbb{R}^{n}\right)$ satisfying $1<p_{-} \leq p_{+}<\infty$. Then there exists a positive constant $C$ such that, for all sequences $\left\{f_{j}\right\}_{j=1}^{\infty}$ of measurable functions,

$$
\left\|\left\{\sum_{j=1}^{\infty}\left[\mathcal{M}\left(f_{j}\right)\right]^{r}\right\}^{1 / r}\right\|_{L^{p(\cdot)\left(\mathbb{R}^{n}\right)}} \leq C\left\|\left(\sum_{j=1}^{\infty}\left|f_{j}\right|^{r}\right)^{1 / r}\right\|_{L^{p(\cdot)}\left(\mathbb{R}^{n}\right)},
$$

where $\mathcal{M}$ denotes the Hardy-Littlewood maximal operator as in 11.3 .

From this, and the fact that, for all balls $B \subset \mathbb{R}^{n}, \beta \in[1, \infty)$ and $r \in(0, \underline{p}), \chi_{\beta B} \leq$ $\beta^{n / r}\left[\mathcal{M}\left(\chi_{B}\right)\right]^{1 / r}$, we obtain the following conclusion, the details being omitted.

Lemma 2.5. Let $p(\cdot) \in C^{\log }\left(\mathbb{R}^{n}\right), r \in(0, p)$ and $\beta \in[1, \infty)$. Then there exists a positive constant $C$ such that, for any sequence $\left\{B_{j}\right\}_{j \in \mathbb{N}}$ of balls of $\mathbb{R}^{n}$,

$$
\left\|\sum_{j \in \mathbb{N}} \chi_{\beta B_{j}}\right\|_{L^{p(\cdot)\left(\mathbb{R}^{n}\right)}} \leq C \beta^{n / r}\left\|\sum_{j \in \mathbb{N}} \chi_{B_{j}}\right\|_{L^{p(\cdot)\left(\mathbb{R}^{n}\right)}} .
$$

The following conclusion is just [40, Remark 4.3].

Lemma 2.6. Let $f \in W H_{\mathrm{atom}}^{p(\cdot), q, s}\left(\mathbb{R}^{n}\right)$. Then

$$
\begin{aligned}
\|f\|_{W H_{\mathrm{atom}}^{p(\cdot), q, s}\left(\mathbb{R}^{n}\right)} & \sim \sup _{i \in \mathbb{Z}} 2^{i}\left\|\left(\sum_{j \in \mathbb{N}} \chi_{B_{i, j}}\right)^{1 / \underline{p}}\right\|_{L^{p(\cdot)\left(\mathbb{R}^{n}\right)}} \sim \sup _{i \in \mathbb{Z}} 2^{i}\left\|\left(\sum_{j \in \mathbb{N}} \chi_{c B_{i, j}}\right)^{1 / \underline{p}}\right\|_{L^{p(\cdot)}\left(\mathbb{R}^{n}\right)} \\
& \sim \sup _{i \in \mathbb{Z}} 2^{i}\left\|\sum_{j \in \mathbb{N}} \chi_{c B_{i, j}}\right\|_{L^{p(\cdot)}\left(\mathbb{R}^{n}\right)} \sim \sup _{i \in \mathbb{Z}} 2^{i}\left\|\sum_{j \in \mathbb{N}} \chi_{B_{i, j}}\right\|_{L^{p(\cdot)\left(\mathbb{R}^{n}\right)}},
\end{aligned}
$$

where $c \in(0,1]$ and the implicit equivalent positive constants are independent of $f$. 
As a variant of [29, Lemma 4.1], the following lemma was obtained in [40, Lemma 4.5]. Lemma 2.7. Let $p(\cdot) \in C^{\log }\left(\mathbb{R}^{n}\right), b \in(0, \underline{p}]$ and $r \in[1, \infty] \cap\left(p_{+}, \infty\right]$. Then there exists a positive constant $C$ such that, for all sequences $\left\{B_{j}\right\}_{j \in \mathbb{N}}$ of balls, numbers $\left\{\lambda_{j}\right\}_{j \in \mathbb{N}} \subset \mathbb{C}$ and measurable functions $\left\{a_{j}\right\}_{j \in \mathbb{N}}$ satisfying that, for each $j \in \mathbb{N}$, supp $a_{j} \subset B_{j}$ and $\left\|a_{j}\right\|_{L^{r}\left(\mathbb{R}^{n}\right)} \leq\left|B_{j}\right|^{1 / r}$, it holds true that

$$
\left\|\left(\sum_{j \in \mathbb{N}}\left|\lambda_{j} a_{j}\right|^{b}\right)^{1 / b}\right\|_{L^{p(\cdot)\left(\mathbb{R}^{n}\right)}} \leq C\left\|\left(\sum_{j \in \mathbb{N}}\left|\lambda_{j} \chi_{B_{j}}\right|^{b}\right)^{1 / b}\right\|_{L^{p(\cdot)}\left(\mathbb{R}^{n}\right)} .
$$

The following lemma is just [22, Proposition 2.4], which, in the case when $s=0$, was first proved by Wilson [33, p. 784].

Lemma 2.8. Let $\alpha \in(0,1], s \in \mathbb{Z}_{+}$and $\epsilon \in(0, \infty)$. Then, for all $f$ satisfying (1.5) and $x \in \mathbb{R}^{n}$, it holds true that

$$
g_{\alpha, s}(f)(x) \sim S_{\alpha, s}(f)(x) \quad \text { and } \quad \widetilde{g}_{(\alpha, \epsilon), s}(f)(x) \sim \widetilde{S}_{(\alpha, \epsilon), s}(f)(x)
$$

with the implicit positive constants independent of $f$.

The following lemma is just [22, Theorem 2.6], which, in the case when $s=0$, was first proved by Wilson [33, p. 775].

Lemma 2.9. Let $\alpha \in(0,1], s \in \mathbb{Z}_{+}$and $\epsilon \in(\max \{\alpha, s\}, \infty)$. Then there exists a positive constant $C$ such that, for all $f$ satisfying 1.5 and $x \in \mathbb{R}^{n}$,

$$
\frac{1}{C} g_{\alpha, s}(f)(x) \leq \widetilde{g}_{(\alpha, \epsilon), s}(f)(x) \leq C g_{\alpha, s}(f)(x) .
$$

The following Lemma 2.10 is a special case of [22, Proposition 3.2].

Lemma 2.10. Let $\alpha \in(0,1], s \in \mathbb{Z}_{+}$and $q \in(1, \infty)$. Then there exists a positive constant $C$ such that, for all measurable functions $f$,

$$
\int_{\mathbb{R}^{n}}\left[g_{\alpha, s}(f)(x)\right]^{q} d x \leq C \int_{\mathbb{R}^{n}}|f(x)|^{q} d x .
$$

Proof of Theorem 1.6. For $\epsilon \in(\max \{\alpha, s\}, \infty)$, by Lemmas 2.8 and 2.9, we see that $g_{\alpha, s}(f), S_{\alpha, s}(f), \widetilde{g}_{(\alpha, \epsilon), s}(f)$ and $\widetilde{S}_{(\alpha, \epsilon), s}(f)$ are pointwise comparable. Thus, to prove this theorem, we only need to consider $g_{\alpha, s}(f)$ in our proof.

Since $\alpha \in(0,1], s \in \mathbb{Z}_{+}$and $p_{-} \in(n /(n+\alpha+s), 1]$, it follows that

$$
s \in\left(\frac{n}{p_{-}}-n-1, \infty\right) \cap \mathbb{Z}_{+} .
$$

Notice that, for all $x \in \mathbb{R}^{n}$,

$$
g(f)(x) \lesssim g_{\alpha, s}(f)(x) .
$$


From this, the facts that $f \in \mathcal{S}^{\prime}\left(\mathbb{R}^{n}\right)$ vanishes weakly at infinity, $g_{\alpha, s}(f) \in W L^{p(\cdot)}\left(\mathbb{R}^{n}\right)$ and Proposition 2.2, we conclude that $f \in W H^{p(\cdot)}\left(\mathbb{R}^{n}\right)$ and

$$
\|f\|_{W H^{p(\cdot)}\left(\mathbb{R}^{n}\right)} \lesssim\|g(f)\|_{W L^{p(\cdot)}\left(\mathbb{R}^{n}\right)} \lesssim\left\|g_{\alpha, s}(f)\right\|_{W L^{p(\cdot)}\left(\mathbb{R}^{n}\right)}
$$

This finishes the proof of Theorem 1.6(i).

It therefore remains to prove Theorem 1.6(ii). Let $f \in W H^{p(\cdot)}\left(\mathbb{R}^{n}\right)$ and $q \in(1, \infty)$. By the fact that $\left.p_{+} \in(0,1], 22.1\right)$ and Proposition 2.1, we know that there exists a sequence $\left\{a_{i, j}\right\}_{i \in \mathbb{Z}, j \in \mathbb{N}}$ of $(p(\cdot), q, s)$-atoms, associated with balls $\left\{B_{i, j}\right\}_{i \in \mathbb{Z}, j \in \mathbb{N}}$, and $\left\{\lambda_{i, j}\right\}_{i \in \mathbb{Z}, j \in \mathbb{N}} \subset \mathbb{C}$ such that

$$
f=\sum_{i \in \mathbb{Z}} \sum_{j \in \mathbb{N}} \lambda_{i, j} a_{i, j} \quad \text { in } \mathcal{S}^{\prime}\left(\mathbb{R}^{n}\right)
$$

and

$$
\|f\|_{W H^{p(\cdot)}\left(\mathbb{R}^{n}\right)}=\|f\|_{W H_{\text {atom }}^{p(\cdot), q, s}\left(\mathbb{R}^{n}\right)} \cdot
$$

For any given $\beta \in(0, \infty)$, we choose $i_{0} \in \mathbb{Z}$ such that $2^{i_{0}} \leq \beta<2^{i_{0}+1}$ and write

$$
f=\sum_{i=-\infty}^{i_{0}-1} \sum_{j \in \mathbb{N}} \lambda_{i, j} a_{i, j}+\sum_{i=i_{0}}^{\infty} \sum_{j \in \mathbb{N}} \lambda_{i, j} a_{i, j}=: f_{1}+f_{2} .
$$

Thus, by Remark 1.2 (iii), we find that

$$
\begin{aligned}
& \quad\left\|\chi_{\left\{x \in \mathbb{R}^{n}: g_{\alpha, s}(f)(x)>\beta\right\}}\right\|_{L^{p(\cdot)}\left(\mathbb{R}^{n}\right)} \\
& \lesssim\left\|\chi_{\left\{x \in \mathbb{R}^{n}: g_{\alpha, s}\left(f_{1}\right)(x)>\beta / 2\right\}}\right\|_{L^{p(\cdot)}\left(\mathbb{R}^{n}\right)}+\left\|\chi_{\left\{x \in A_{i_{0}}: g_{\alpha, s}\left(f_{2}\right)(x)>\beta / 2\right\}}\right\|_{L^{p(\cdot)}\left(\mathbb{R}^{n}\right)} \\
& \quad+\left\|\chi_{\left\{x \in\left(A_{i_{0}}\right)^{\complement}: g_{\alpha, s}\left(f_{2}\right)(x)>\beta / 2\right\}}\right\|_{L^{p(\cdot)\left(\mathbb{R}^{n}\right)}}=: \mathrm{I}_{1}+\mathrm{I}_{2}+\mathrm{I}_{3},
\end{aligned}
$$

where $A_{i_{0}}:=\bigcup_{i=i_{0}}^{\infty} \bigcup_{j \in \mathbb{N}}\left(2 B_{i, j}\right)$.

It is easy to see that

$$
\begin{aligned}
\mathrm{I}_{1} \lesssim & \left\|\chi_{\left\{x \in \mathbb{R}^{n}: \sum_{i=-\infty}^{i_{0}-1} \sum_{j \in \mathbb{N}} \lambda_{i, j} g_{\alpha, s}\left(a_{i, j}\right)(x) \chi_{2 B_{i, j}}(x)>\beta / 4\right\}}\right\|_{L^{p(\cdot)}\left(\mathbb{R}^{n}\right)} \\
& +\left\|\chi_{\left\{x \in \mathbb{R}^{n}: \sum_{i=-\infty}^{i_{0}-1} \sum_{j \in \mathbb{N}} \lambda_{i, j} g_{\alpha, s}\left(a_{i, j}\right)(x) \chi_{\left(2 B_{i, j}\right)^{\complement}}(x)>\beta / 4\right\}}\right\|_{L^{p(\cdot)}\left(\mathbb{R}^{n}\right)}=: \mathrm{I}_{1,1}+\mathrm{I}_{1,2} .
\end{aligned}
$$

To estimate $\mathrm{I}_{1,1}$, for any $b \in(0, \underline{p})$, let $q_{1} \in(1, \min \{q, 1 / b\})$ and $a \in\left(0,1-1 / q_{1}\right)$. Then, by the Hölder inequality, we find that, for all $x \in \mathbb{R}^{n}$,

$$
\begin{aligned}
& \sum_{i=-\infty}^{i_{0}-1} \sum_{j \in \mathbb{N}} \lambda_{i, j} g_{\alpha, s}\left(a_{i, j}\right)(x) \chi_{2 B_{i, j}}(x) \\
\leq & \left(\sum_{i=-\infty}^{i_{0}-1} 2^{i a q_{1}^{\prime}}\right)^{1 / q_{1}^{\prime}}\left\{\sum_{i=-\infty}^{i_{0}-1} 2^{-i a q_{1}}\left[\sum_{j \in \mathbb{N}} \lambda_{i, j} g_{\alpha, s}\left(a_{i, j}\right)(x) \chi_{2 B_{i, j}}(x)\right]^{q_{1}}\right\}^{1 / q_{1}}
\end{aligned}
$$




$$
=\frac{2^{i_{0} a}}{\left(2^{a q_{1}^{\prime}}-1\right)^{1 / q_{1}^{\prime}}}\left\{\sum_{i=-\infty}^{i_{0}-1} 2^{-i a q_{1}}\left[\sum_{j \in \mathbb{N}} \lambda_{i, j} g_{\alpha, s}\left(a_{i, j}\right)(x) \chi_{2 B_{i, j}}(x)\right]^{q_{1}}\right\}^{1 / q_{1}},
$$

where $q_{1}^{\prime}$ denotes the conjugate exponent of $q_{1}$, namely, $1 / q_{1}+1 / q_{1}^{\prime}=1$. From this, the fact that $q_{1} b<1$, the value of $\lambda_{i, j}$, Remark 1.2 (iii) and Lemma 1.3 , we deduce that

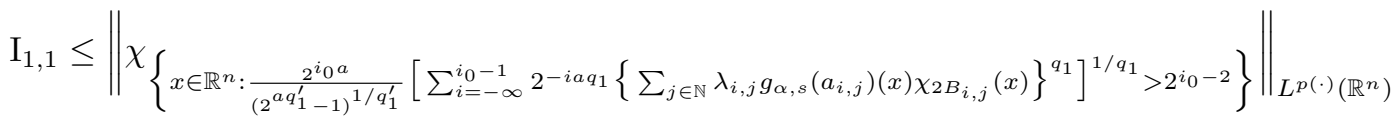

$$
\begin{aligned}
& \lesssim 2^{-i_{0} q_{1}(1-a)}\left\|\sum_{i=-\infty}^{i_{0}-1} 2^{-i a q_{1}}\left[\sum_{j \in \mathbb{N}} \lambda_{i, j} g_{\alpha, s}\left(a_{i, j}\right) \chi_{2 B_{i, j}}\right]^{q_{1}}\right\|_{L^{p(\cdot)}\left(\mathbb{R}^{n}\right)} \\
& \lesssim 2^{-i_{0} q_{1}(1-a)}\left\|\sum_{i=-\infty}^{i_{0}-1} 2^{(1-a) i q_{1} b} \sum_{j \in \mathbb{N}}\left[\left\|\chi_{B_{i, j}}\right\|_{L^{p(\cdot)}\left(\mathbb{R}^{n}\right)} g_{\alpha, s}\left(a_{i, j}\right) \chi_{2 B_{i, j}}\right]^{q_{1} b}\right\|_{L^{p(\cdot) / b}\left(\mathbb{R}^{n}\right)}^{1 / b} \\
& \lesssim 2^{-i_{0} q_{1}(1-a)}\left[\sum_{i=-\infty}^{i_{0}-1} 2^{(1-a) i q_{1} b}\left\|\left\{\sum_{j \in \mathbb{N}}\left[\left\|\chi_{B_{i, j}}\right\|_{L^{p(\cdot)}\left(\mathbb{R}^{n}\right)} g_{\alpha, s}\left(a_{i, j}\right) \chi_{2 B_{i, j}}\right]^{q_{1} b}\right\}^{1 / b}\right\|_{L^{p(\cdot)}\left(\mathbb{R}^{n}\right)}^{b}\right]^{1 / b} .
\end{aligned}
$$

Now let $r:=q / q_{1}$. Then $r \in(1, \infty)$ and, by Lemma 2.10 , we find that, for all $i \in \mathbb{Z}$ and $j \in \mathbb{N}$,

$$
\begin{aligned}
\left\|\left[\left\|\chi_{B_{i, j}}\right\|_{L^{p(\cdot)}\left(\mathbb{R}^{n}\right)} g_{\alpha, s}\left(a_{i, j}\right) \chi_{2 B_{i, j}}\right]^{q_{1}}\right\|_{L^{r}\left(\mathbb{R}^{n}\right)} & \lesssim\left\|\chi_{B_{i, j}}\right\|_{L^{p(\cdot)}\left(\mathbb{R}^{n}\right)}^{q_{1}}\left\|g_{\alpha, s}\left(a_{i, j}\right) \chi_{2 B_{i, j}}\right\|_{L^{q}\left(\mathbb{R}^{n}\right)}^{q_{1}} \\
& \lesssim\left|B_{i, j}\right|^{1 / r} .
\end{aligned}
$$

Therefore, by Lemmas 2.7, 2.5 and 2.6, we conclude that

$$
\begin{aligned}
\mathrm{I}_{1,1} & \lesssim 2^{-i_{0} q_{1}(1-a)}\left[\sum_{i=-\infty}^{i_{0}-1} 2^{(1-a) i q_{1} b}\left\|\left(\sum_{j \in \mathbb{N}} \chi_{2 B_{i, j}}\right)^{1 / b}\right\|_{L^{p(\cdot)}\left(\mathbb{R}^{n}\right)}^{b}\right]^{1 / b} \\
& \lesssim 2^{-i_{0} q_{1}(1-a)}\left[\sum_{i=-\infty}^{i_{0}-1} 2^{(1-a) i q_{1} b}\left\|\left(\sum_{j \in \mathbb{N}} \chi_{c B_{i, j}}\right)^{1 / b}\right\|_{L^{p(\cdot)}\left(\mathbb{R}^{n}\right)}^{b}\right]^{1 / b} \\
& \lesssim 2^{-i_{0} q_{1}(1-a)}\left\{\sum_{i=-\infty}^{i_{0}-1} 2^{\left[(1-a) q_{1}-1\right] i b}\right\}^{1 / b} \sup _{i \in \mathbb{Z}} 2^{i}\left\|\sum_{j \in \mathbb{N}} \chi_{B_{i, j}}\right\|_{L^{p(\cdot)}\left(\mathbb{R}^{n}\right)} \\
& \sim \beta^{-1}\|f\|_{W H_{\text {atom }}^{p(\cdot), q, s}\left(\mathbb{R}^{n}\right)},
\end{aligned}
$$

which implies that

$$
\beta \mathrm{I}_{1,1} \lesssim\|f\|_{W H_{\text {atom }}^{p(\cdot), q, s}\left(\mathbb{R}^{n}\right)}
$$

To deal with $\mathrm{I}_{1,2}$, we need some estimates on $g_{\alpha, s}\left(a_{i, j}\right)$. Let $\phi \in \mathcal{C}_{\alpha, s}\left(\mathbb{R}^{n}\right)$ and, for any $i \in \mathbb{Z}$ and $j \in \mathbb{N}, B_{i, j}:=B\left(x_{i, j}, r_{i, j}\right)$ with some $x_{i, j} \in \mathbb{R}^{n}$ and $r_{i, j} \in(0, \infty)$. From the vanishing moment of $a_{i, j}$, Taylor's remainder theorem, (1.4) and the Hölder inequality, we 
deduce that, for all $i \in \mathbb{Z} \cap\left(-\infty, i_{0}-1\right], j \in \mathbb{N}, t \in(0, \infty)$ and $x \in\left(2 B_{i, j}\right)^{\complement}$,

$$
\begin{aligned}
\left|a_{i, j} * \phi_{t}(x)\right| & =\left|\int_{B_{i, j}} a_{i, j}(y)\left[\phi\left(\frac{x-y}{t}\right)-\sum_{|\gamma| \leq s} \frac{D^{\gamma} \phi\left(\frac{x-x_{i, j}}{t}\right)}{\gamma !}\left(\frac{x_{i, j}-y}{t}\right)^{\gamma}\right] \frac{d y}{t^{n}}\right| \\
& \sim\left|\int_{B_{i, j}} a_{i, j}(y)\left\{\sum_{|\gamma|=s}\left[D^{\gamma} \phi(\xi)-D^{\gamma} \phi\left(\frac{x-x_{i, j}}{t}\right)\right]\left(\frac{x_{i, j}-y}{t}\right)^{\gamma}\right\} \frac{d y}{t^{n}}\right| \\
& \sim\left|\int_{B_{i, j}} a_{i, j}(y)\left\{\sum_{|\gamma|=s}\left(\frac{\theta\left|x_{i, j}-y\right|}{t}\right)^{\alpha}\left(\frac{x_{i, j}-y}{t}\right)^{\gamma}\right\} \frac{d y}{t^{n}}\right| \\
& \lesssim \frac{r_{i, j}^{\alpha+s}}{t^{n+\alpha+s}}\left[\int_{B_{i, j}}\left|a_{i, j}(y)\right|^{q} d y\right]^{1 / q}\left(\int_{B_{i, j}} 1 d y\right)^{1 / q^{\prime}} \\
& \lesssim\left\|\chi_{B_{i, j}}\right\|_{L^{p(\cdot)}\left(\mathbb{R}^{n}\right)}^{-1}\left(\frac{r_{i, j}}{t}\right)^{n+\alpha+s},
\end{aligned}
$$

where $\xi=\left[\left(x-x_{i, j}\right)+\theta\left(x_{i, j}-y\right)\right] / t$ for some $\theta \in(0,1)$.

Notice that $\operatorname{supp} \phi \subset\left\{x \in \mathbb{R}^{n}:|x| \leq 1\right\}$. If $x \in\left(2 B_{i, j}\right)^{\complement}$ and $a_{i, j} * \phi_{t}(x) \neq 0$, then, there exists a $y \in B_{i, j}$ such that $|x-y| / t \leq 1$ and hence $t \geq|x-y| \geq\left|x-x_{i, j}\right|-\left|x_{i, j}-y\right| \geq$ $\left|x-x_{i, j}\right| / 2$. From this and 2.6 , we deduce that

$$
\begin{aligned}
g_{\alpha, s}\left(a_{i, j}\right)(x) & =\left\{\int_{0}^{\infty}\left[\sup _{\phi \in \mathcal{C}_{\alpha, s}\left(\mathbb{R}^{n}\right)}\left|a_{i, j} * \phi_{t}(x)\right|\right]^{2} \frac{d t}{t}\right\}^{1 / 2} \\
& \lesssim \frac{r_{i, j}^{n+\alpha+s}}{\left\|\chi_{B_{i, j}}\right\|_{L^{p(\cdot)}\left(\mathbb{R}^{n}\right)}}\left\{\int_{\left|x-x_{i, j}\right| / 2}^{\infty} t^{-2(n+\alpha+s)-1} d t\right\}^{1 / 2} \\
& \lesssim\left\|\chi_{B_{i, j}}\right\|_{L^{p(\cdot)}\left(\mathbb{R}^{n}\right)}^{-1}\left(\frac{r_{i, j}}{\left|x-x_{i, j}\right|}\right)^{n+\alpha+s} \lesssim \frac{\left[\mathcal{M}\left(\chi_{B_{i, j}}\right)(x)\right]^{(n+\alpha+s) / n}}{\left\|\chi_{B_{i, j}}\right\|_{L^{p(\cdot)}\left(\mathbb{R}^{n}\right)}}
\end{aligned}
$$

By this, the Hölder inequality, the value of $\lambda_{i, j}$, Remark 1.2(iii) and Lemmas 1.3, 2.4, 2.5 and 2.6. we find that, for any $b \in(0, n /(n+\alpha+s)), q_{2} \in(n /[(n+\alpha+s) b], 1 / b)$ and $a \in\left(0,1-1 / q_{2}\right)$,

$$
\begin{aligned}
& \mathrm{I}_{1,2} \leq\left\|\chi_{\left\{x \in \mathbb{R}^{n}: \frac{2^{i} 0^{a}}{\left(2^{\left.a q_{2}^{\prime}-1\right)^{1 / q_{2}^{\prime}}}\right.}\left\{\sum_{i=-\infty}^{i_{0}-1} 2^{-i a q_{2}}\left[\sum_{j \in \mathbb{N}} \lambda_{i, j} g_{\alpha, s}\left(a_{i, j}\right)(x) \chi_{\left(2 B_{i, j}\right)} \mathrm{C}(x)\right]^{q_{2}}\right\}^{1 / q_{2}}>2^{i_{0}-2}\right\}}\right\|_{L^{p(\cdot)}\left(\mathbb{R}^{n}\right)} \\
& \lesssim 2^{-i_{0} q_{2}(1-a)}\left\|\sum_{i=-\infty}^{i_{0}-1} 2^{-i a q_{2}}\left[\sum_{j \in \mathbb{N}} \lambda_{i, j} g_{\alpha, s}\left(a_{i, j}\right) \chi_{\left(2 B_{i, j}\right)^{\mathrm{C}}}\right]^{q_{2}}\right\|_{L^{p(\cdot)}\left(\mathbb{R}^{n}\right)} \\
& \lesssim 2^{-i_{0} q_{2}(1-a)}\left\{\sum_{i=-\infty}^{i_{0}-1} 2^{(1-a) i q_{2} b}\left\|\sum_{j \in \mathbb{N}}\left[\mathcal{M}\left(\chi_{B_{i, j}}\right)\right]^{(n+\alpha+s) q_{2} b / n}\right\|_{L^{p(\cdot) / b}\left(\mathbb{R}^{n}\right)}\right\}^{1 / b}
\end{aligned}
$$

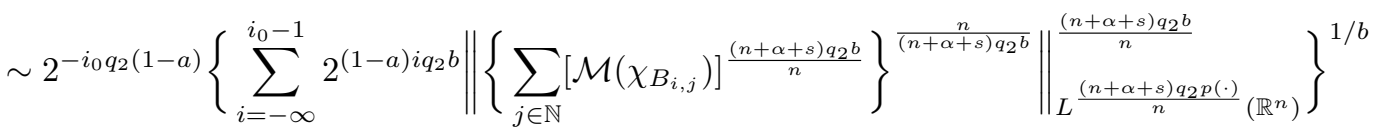

$$
\begin{aligned}
& \lesssim 2^{-i_{0} q_{2}(1-a)}\left[\sum_{i=-\infty}^{i_{0}-1} 2^{(1-a) i q_{2} b}\left\|\sum_{j \in \mathbb{N}} \chi_{B_{i, j}}\right\|_{L^{p(\cdot) / b}\left(\mathbb{R}^{n}\right)}\right]^{1 / b}
\end{aligned}
$$




$$
\begin{aligned}
& \lesssim 2^{-i_{0} q_{2}(1-a)}\left\{\sum_{i=-\infty}^{i_{0}-1} 2^{\left[(1-a) q_{2}-1\right] i b} 2^{i b}\left\|\left(\sum_{j \in \mathbb{N}} \chi_{c B_{i, j}}\right)^{1 / b}\right\|_{L^{p(\cdot)}\left(\mathbb{R}^{n}\right)}^{b}\right\}^{1 / b} \\
& \lesssim \beta^{-1} \sup _{i \in \mathbb{Z}} 2^{i}\left\|\sum_{j \in \mathbb{N}} \chi_{B_{i, j}}\right\|_{L^{p(\cdot)}\left(\mathbb{R}^{n}\right)} \sim \beta^{-1}\|f\|_{W H_{\text {atom }}^{p(\cdot), q, s}\left(\mathbb{R}^{n}\right)},
\end{aligned}
$$

that is

$$
\beta \mathrm{I}_{1,2} \lesssim\|f\|_{W H_{\text {atom }}^{p(\cdot), q, s}\left(\mathbb{R}^{n}\right)} \cdot
$$

By this, combined with (2.4) and (2.5), we conclude that

$$
\beta \mathrm{I}_{1} \lesssim\|f\|_{W H_{\mathrm{atom}}^{p(\cdot), q, s}\left(\mathbb{R}^{n}\right)} .
$$

For $\mathrm{I}_{2}$, we choose $r_{1} \in[1 / \underline{p}, \infty)$. Then, by Remark $1.2(\mathrm{iii})$, Lemmas 1.3 , 2.5 and 2.6. we conclude that

$$
\begin{aligned}
\mathrm{I}_{2} & \leq\left\|\chi_{A_{i_{0}}}\right\|_{L^{p(\cdot)\left(\mathbb{R}^{n}\right)}} \leq\left\|\sum_{i=i_{0}}^{\infty} \sum_{j \in \mathbb{N}} \chi_{2 B_{i, j}}\right\|_{L^{p(\cdot)\left(\mathbb{R}^{n}\right)}} \lesssim\left\|\sum_{i=i_{0}}^{\infty} \sum_{j \in \mathbb{N}} \chi_{B_{i, j}}\right\|_{L^{p(\cdot)}\left(\mathbb{R}^{n}\right)} \\
& \lesssim\left[\sum_{i=i_{0}}^{\infty}\left\|\sum_{j \in \mathbb{N}} \chi_{B_{i, j}}\right\|_{L^{p(\cdot)\left(\mathbb{R}^{n}\right)}}^{1 / r_{1}}\right]^{r_{1}} \sim\left\{\sum_{i=i_{0}}^{\infty} 2^{-i / r_{1}}\left[2^{i}\left\|\sum_{j \in \mathbb{N}} \chi_{B_{i, j}}\right\|_{L^{p(\cdot)\left(\mathbb{R}^{n}\right)}}\right]^{1 / r_{1}}\right\}^{r_{1}} \\
& \lesssim \sup _{i \in \mathbb{Z}} 2^{i}\left\|\sum_{j \in \mathbb{N}} \chi_{B_{i, j}}\right\|_{L^{p(\cdot)\left(\mathbb{R}^{n}\right)}}\left(\sum_{i=i_{0}}^{\infty} 2^{-i / r_{1}}\right)^{r_{1}} \sim \beta^{-1}\|f\|_{W H_{\text {atom }}^{p(\cdot), q, s}\left(\mathbb{R}^{n}\right)},
\end{aligned}
$$

which implies that

$$
\beta \mathrm{I}_{2} \lesssim\|f\|_{W H_{\text {atom }}^{p(\cdot), q, s}\left(\mathbb{R}^{n}\right)} \cdot
$$

For $\mathrm{I}_{3}$, since $\underline{p} \in(n /(n+\alpha+s), 1]$, it follows that there exists $r_{2} \in(0, \infty)$ such that $r_{2} \in(n /[\underline{p}(n+\alpha+s)], 1)$. By this, the value of $\lambda_{i, j},(2.7)$, Remark 1.2 (iii) and Lemmas 1.3 . 2.4 and 2.6 , we find that

$$
\begin{aligned}
& \mathrm{I}_{3} \leq\left\|\chi_{\left\{x \in\left(A_{i_{0}}\right)^{\mathrm{C}}: \sum_{i=i_{0}}^{\infty} \sum_{j \in \mathbb{N}} \lambda_{i, j} g_{\alpha, s}\left(a_{i, j}\right)(x)>\beta\right\}}\right\|_{L^{p(\cdot)}\left(\mathbb{R}^{n}\right)} \\
& \leq \beta^{-r_{2}}\left\|\sum_{i=i_{0}}^{\infty} \sum_{j \in \mathbb{N}}\left[\lambda_{i, j} g_{\alpha, s}\left(a_{i, j}\right)\right]^{r_{2}} \chi_{\left(A_{i_{0}}\right)} \mathrm{C}\right\|_{L^{p(\cdot)}\left(\mathbb{R}^{n}\right)} \\
& \leq \beta^{-r_{2}}\left[\sum_{i=i_{0}}^{\infty}\left\|\left\{\sum_{j \in \mathbb{N}}\left[\lambda_{i, j} g_{\alpha, s}\left(a_{i, j}\right)\right]^{r_{2}} \chi_{\left(A_{i_{0}}\right)^{\complement}}\right\}^{\frac{n}{r_{2}(n+\alpha+s)}}\right\|_{L}^{\frac{r_{2}(n+\alpha+s)}{n} p(\cdot)\left(\mathbb{R}^{n}\right)}\right]^{\frac{r_{2}(n+\alpha+s)}{n}} \\
& \lesssim \beta^{-r_{2}}\left\{\sum_{i=i_{0}}^{\infty} 2^{\frac{i n}{n+\alpha+s}}\left\|\left\{\sum_{j \in \mathbb{N}}\left[\mathcal{M}\left(\chi_{B_{i, j}}\right)\right]^{\frac{r_{2}(n+\alpha+s)}{n}}\right\}^{\frac{n}{r_{2}(n+\alpha+s)}}\right\|_{L}{ }_{L}^{\frac{r_{2}(n+\alpha+s)}{n} p(\cdot)\left(\mathbb{R}^{n}\right)}\right\}^{\frac{r_{2}(n+\alpha+s)}{n}} \\
& \lesssim \beta^{-r_{2}}\left[\sum_{i=i_{0}}^{\infty} 2^{\frac{i n}{n+\alpha+s}}\left\|\sum_{j \in \mathbb{N}} \chi_{B_{i, j}}\right\|_{L^{p(\cdot)}\left(\mathbb{R}^{n}\right)}^{\frac{n}{r_{2}(n+\alpha+s)}}\right]^{\frac{r_{2}(n+\alpha+s)}{n}}
\end{aligned}
$$




$$
\begin{aligned}
& \lesssim \sup _{i \in \mathbb{Z}} 2^{i}\left\|\sum_{j \in \mathbb{N}} \chi_{B_{i, j}}\right\|_{L^{p(\cdot)\left(\mathbb{R}^{n}\right)}} \beta^{-r_{2}}\left[\sum_{i=i_{0}}^{\infty} 2^{\frac{i n}{n+\alpha+s}} 2^{-\frac{i n}{r_{2}(n+\alpha+s)}}\right]^{\frac{r_{2}(n+\alpha+s)}{n}} \\
& \sim \beta^{-1}\|f\|_{W H_{\text {atom }}^{p(\cdot), q, s}\left(\mathbb{R}^{n}\right)}
\end{aligned}
$$

namely,

$$
\beta \mathrm{I}_{3} \lesssim\|f\|_{W H_{\text {atom }}^{p(\cdot), q, s}\left(\mathbb{R}^{n}\right)} \cdot
$$

Combining with (2.3), 2.8), 2.9), 2.10) and (2.2), we obtain

$$
\begin{aligned}
\left\|g_{\alpha, s}(f)\right\|_{W L^{p(\cdot)}\left(\mathbb{R}^{n}\right)} & =\sup _{\beta \in(0, \infty)} \beta\left\|\chi_{\left\{x \in \mathbb{R}^{n}: g_{\alpha, s}(f)(x)>\beta\right\}}\right\|_{L^{p(\cdot)}\left(\mathbb{R}^{n}\right)} \\
& \lesssim \sup _{\beta \in(0, \infty)} \beta\left(\mathrm{I}_{1}+\mathrm{I}_{2}+\mathrm{I}_{3}\right) \lesssim\|f\|_{W H_{\mathrm{atom}}^{p(\cdot), q, s}\left(\mathbb{R}^{n}\right)} \sim\|f\|_{W H^{p(\cdot)}\left(\mathbb{R}^{n}\right)}
\end{aligned}
$$

which finishes the proof of Theorem 1.6(ii).

Proof of Theorem 1.7. Let $f \in \mathcal{S}^{\prime}\left(\mathbb{R}^{n}\right), f$ vanishes weakly at infinity and $g_{\lambda, \alpha, s}^{*}(f) \in$ $W L^{p(\cdot)}\left(\mathbb{R}^{n}\right)$. Since $\alpha \in(0,1], s \in \mathbb{Z}_{+}, p_{-} \in(n /(n+\alpha+s), 1]$ and $\lambda \in(3+2(\alpha+s) / n, \infty)$, it follows that

$$
\lambda>3+\frac{2(\alpha+s)}{n}>3+\frac{2\left(1-p_{-}\right)}{p_{-}}=1+\frac{2}{p_{-}}=1+\frac{2}{\min \left\{p_{-}, 2\right\}} .
$$

From this, the fact that for all $x \in \mathbb{R}^{n}, g_{\lambda}^{*}(f)(x) \lesssim g_{\lambda, \alpha, s}^{*}(f)(x) \lesssim \widetilde{g}_{\lambda,(\alpha, \epsilon), s}^{*}(f)(x)$ and Proposition 2.3. we conclude that $f \in W H^{p(\cdot)}\left(\mathbb{R}^{n}\right)$ and

$$
\|f\|_{W H^{p(\cdot)\left(\mathbb{R}^{n}\right)}} \lesssim\left\|g_{\lambda}^{*}(f)\right\|_{W L^{p(\cdot)}\left(\mathbb{R}^{n}\right)} \lesssim\left\|g_{\lambda, \alpha, s}^{*}(f)\right\|_{W L^{p(\cdot)}\left(\mathbb{R}^{n}\right)} \lesssim\left\|\widetilde{g}_{\lambda,(\alpha, \epsilon), s}^{*}(f)\right\|_{W L^{p(\cdot)\left(\mathbb{R}^{n}\right)}} .
$$

This finishes the proof of Theorem 1.7(i).

It therefore remains to prove Theorem $1.7\left(\right.$ ii). Let $f \in W H^{p(\cdot)}\left(\mathbb{R}^{n}\right)$. Then, by an argument similar to that used in 45 , p. 1566], we conclude that for all $\alpha \in(0,1], s \in \mathbb{Z}_{+}$, $\epsilon \in(\max \{\alpha, s\}, \infty), \lambda \in(3+2(\alpha+s) / n, \infty)$ and $x \in \mathbb{R}^{n}$,

$$
\widetilde{g}_{\lambda,(\alpha, \epsilon), s}^{*}(f)(x) \lesssim \widetilde{S}_{(\alpha, \epsilon), s}(f)(x),
$$

from this and Theorem 1.6(ii), we deduce that

$$
\begin{aligned}
\left\|g_{\lambda, \alpha, s}^{*}(f)\right\|_{W L^{p(\cdot)}\left(\mathbb{R}^{n}\right)} & \lesssim\left\|\widetilde{g}_{\lambda,(\alpha, \epsilon), s}^{*}(f)\right\|_{W L^{p(\cdot)}\left(\mathbb{R}^{n}\right)} \\
& \lesssim\left\|\widetilde{S}_{(\alpha, \epsilon), s}(f)\right\|_{W L^{p(\cdot)}\left(\mathbb{R}^{n}\right)} \lesssim\|f\|_{W H^{p(\cdot)}\left(\mathbb{R}^{n}\right)},
\end{aligned}
$$

which completes the proof of Theorem 1.7(ii). 


\section{References}

[1] W. Abu-Shammala and A. Torchinsky, The Hardy-Lorentz spaces $H^{p, q}\left(\mathbb{R}^{n}\right)$, Studia Math. 182 (2007), no. 3, 283-294.

[2] N. Aguilera and C. Segovia, Weighted norm inequalities relating the $g_{\lambda}^{*}$ and the area functions, Studia Math. 61 (1977), no. 3, 293-303.

[3] A. Almeida and A. Caetano, Atomic and molecular decompositions in variable exponent 2-microlocal spaces and applications, J. Funct. Anal. 270 (2016), no. 5, 18881921.

[4] A. Almeida and P. Hästö, Besov spaces with variable smoothness and integrability, J. Funct. Anal. 258 (2010), no. 5, 1628-1655.

[5] J. Álvarez, $H^{p}$ and weak $H^{p}$ continuity of Calderón-Zygmund type operators, in: Fourier Analysis (Orono, ME, 1992), 17-34, Lecture Notes in Pure and Appl. Math. 157, Dekker, New York, 1994.

[6] _ Continuity properties for linear commutators of Calderón-Zygmund operators, Collect. Math. 49 (1998), no. 1, 17-31.

[7] Z. Birnbaum and W. Orlicz, Über die Verallgemeinerung des Begriffes der zueinander konjugierten Potenzen, Studia Math. 3 (1931), no. 1, 1-67.

[8] D. V. Cruz-Uribe and A. Fiorenza, Variable Lebesgue Spaces: Foundations and harmonic analysis, Applied and Numerical Harmonic Analysis, Birkhäuser/Springer, Heidelberg, 2013.

[9] D. Cruz-Uribe, A. Fiorenza, J. M. Martell and C. Pérez, The boundedness of classical operators on variable $L^{p}$ spaces, Ann. Acad. Sci. Fenn. Math. 31 (2006), no. 1, 239264.

[10] D. Cruz-Uribe and L.-A. D. Wang, Variable Hardy spaces, Indiana Univ. Math. J. 63 (2014), no. 2, 447-493.

[11] L. Diening, P. Harjulehto, P. Hästö and M. Růžička, Lebesgue and Sobolev Spaces with Variable Exponents, Lecture Notes in Mathematics 2017, Springer, Heidelberg, 2011.

[12] L. Diening, P. Hästö and S. Roudenko, Function spaces of variable smoothness and integrability, J. Funct. Anal. 256 (2009), no. 6, 1731-1768. 
[13] Y. Ding, S. Lu and S. Shao, Integral operators with variable kernels on weak Hardy spaces, J. Math. Anal. Appl. 317 (2006), no. 1, 127-135.

[14] C. Fefferman, N. M. Rivière and Y. Sagher, Interpolation between $H^{p}$ spaces: the real method, Trans. Amer. Math. Soc. 191 (1974), 75-81.

[15] C. Fefferman and E. M. Stein, $H^{p}$ spaces of several variables, Acta Math. 129 (1972), no. 3-4, 137-193.

[16] R. Fefferman and F. Soria, The space Weak $H^{1}$, Studia Math. 85 (1986), no. 1, 1-16.

[17] G. B. Folland and E. M. Stein, Hardy Spaces on Homogeneous Groups, Mathematical Notes 28, Princeton University Press, Princeton, N.J., 1982.

[18] J. Huang and Y. Liu, Some characterizations of weighted Hardy spaces, J. Math. Anal. Appl. 363 (2010), no. 1, 121-127.

[19] M. Izuki, E. Nakai and Y. Sawano, Function spaces with variable exponents-an introduction, Sci. Math. Jpn. 77 (2014), no. 2, 187-315.

[20] A. K. Lerner, Sharp weighted norm inequalities for Littlewood-Paley operators and singular integrals, Adv. Math. 226 (2011), no. 5, 3912-3926.

[21] _ On sharp aperture-weighted estimates for square functions, J. Fourier Anal. Appl. 20 (2014), no. 4, 784-800.

[22] Y. Liang and D. Yang, Intrinsic square function characterizations of Musielak-Orlicz Hardy spaces, Trans. Amer. Math. Soc. 367 (2015), no. 5, 3225-3256.

[23] H.-p. Liu, The weak $H^{p}$ spaces on homogenous groups, in: Harmonic Analysis (Tianjin, 1988), 113-118, Lecture Notes in Mathematics 1494, Springer, Berlin, 1991.

[24] J. Liu, D. Yang and W. Yuan, Anisotropic variable Hardy-Lorentz spaces and their real interpolation, J. Math. Anal. Appl. 456 (2017), no. 1, 356-393.

[25] S. Z. Lu, Four Lectures on Real $H^{p}$ Spaces, World Scientific, River Edge, NJ, 1995.

[26] E. Nakai and Y. Sawano, Hardy spaces with variable exponents and generalized Campanato spaces, J. Funct. Anal. 262 (2012), no. 9, 3665-3748.

[27] W. Orlicz, Über konjugierte Exponentenfolgen, Studia Math. 3 (1931), no. 1, 200-211.

[28] T. Quek and D. Yang, Calderón-Zygmund-type operators on weighted weak Hardy spaces over $\mathbb{R}^{n}$, Acta Math. Sin. (Engl. Ser.) 16 (2000), no. 1, 141-160. 
[29] Y. Sawano, Atomic decompositions of Hardy spaces with variable exponents and its application to bounded linear operators, Integral Equations Operator Theory 77 (2013), no. $1,123-148$.

[30] H. Wang, Boundedness of intrinsic square functions on the weighted weak Hardy spaces, Integral Equations Operator Theory 75 (2013), no. 1, 135-149.

[31] _ Boundedness of several integral operators with bounded variable kernels on Hardy and weak Hardy spaces, Internat. J. Math. 24 (2013), no. 12, 1350095, 22 pp.

[32] H. Wang and H. Liu, The intrinsic square function characterizations of weighted Hardy spaces, Illinois J. Math. 56 (2012), no. 2, 367-381.

[33] M. Wilson, The intrinsic square function, Rev. Mat. Iberoam. 23 (2007), no. 3, 771791.

[34] _ Weighted Littlewood-Paley Theory and Exponential-square Integrability, Lecture Notes in Mathematics 1924, Springer, Berlin, 2008.

[35] _ How fast and in what sense(s) does the Calderón reproducing formula converge? J. Fourier Anal. Appl. 16 (2010), no. 5, 768-785.

[36] Convergence and stability of the Calderón reproducing formula in $H^{1}$ and BMO, J. Fourier Anal. Appl. 17 (2011), no. 5, 801-820.

[37] L. Wu, D. Zhou, C. Zhuo and Y. Jiao, Riesz transform characterizations of variable Hardy-Lorentz spaces, Rev. Mat. Complut. 31 (2018), no. 3, 747-780.

[38] J. Xu, Variable Besov and Triebel-Lizorkin spaces, Ann. Acad. Sci. Fenn. Math. 33 (2008), no. 2, 511-522.

[39] X. Yan, Intrinsic square function characterizations of weak Musielak-Orlicz Hardy spaces, Accepted.

[40] X. Yan, D. Yang, W. Yuan and C. Zhuo, Variable weak Hardy spaces and their applications, J. Funct. Anal. 271 (2016), no. 10, 2822-2887.

[41] D. Yang, W. Yuan and C. Zhuo, A survey on some variable function spaces, in: Function Spaces and Inequalities, 299-335, Springer Proc. Math. Stat. 206, Springer, Singapore, 2017.

[42] D. Yang, C. Zhuo and E. Nakai, Characterizations of variable exponent Hardy spaces via Riesz transforms, Rev. Mat. Complut. 29 (2016), no. 2, 245-270. 
[43] D. Yang, C. Zhuo and W. Yuan, Triebel-Lizorkin type spaces with variable exponents, Banach J. Math. Anal. 9 (2015), no. 4, 146-202.

[44] _ Besov-type spaces with variable smoothness and integrability, J. Funct. Anal. 269 (2015), no. 6, 1840-1898.

[45] C. Zhuo, D. Yang and Y. Liang, Intrinsic square function characterizations of Hardy spaces with variable exponents, Bull. Malays. Math. Sci. Soc. 39 (2016), no. 4, 15411577.

[46] C. Zhuo, D. Yang and W. Yuan, Interpolation between $H^{p(\cdot)}\left(\mathbb{R}^{n}\right)$ and $L^{\infty}\left(\mathbb{R}^{n}\right)$ : real method, J. Geom. Anal. 28 (2018), no. 3, 2288-2311.

Xianjie Yan

Department of Basic Course, Zhengzhou University of Science and Technology,

Zhengzhou, Henan 450064, P. R. China

E-mail address: xianjieyan@mail.bnu.edu.cn 